\title{
Sangrado de origen ginecológico
}

\section{Gynaecological bleeding}

\author{
I. Jiménez Ubieto, A. Zornoza, O. Tarrío
}

\section{RESUMEN}

El sangrado genital es la alteración ginecológica más frecuente en las mujeres en edad reproductiva siendo, en los países occidentales, una de las causas más frecuentes de anemia y que puede ser motivo de absentismo laboral. Ante una paciente con sangrado en el Servicio de Urgencias lo primero habrá que valorar la repercusión hemodinámica, adoptando las medidas necesarias. Se realizará una anamnesis y exploración detalladas para establecer el origen. Cuando aparece sangrado en el área genital generalmente se piensa en un origen uterino, pero no siempre es así y habrá que descartar origen en el tracto genital inferior, en el tracto genital superior $\mathrm{u}$ otro origen extraginecológico en el área digestiva y/o urológica. Con el diagnóstico se valorará el tratamiento oportuno y la necesidad o no de ingreso hospitalario.

Palabras clave. Metrorragia. Hemorragia uterina disfuncional. Urgencias en ginecología.

\begin{abstract}
Genital bleeding is the most frequent gynaecological alteration in women of reproductive age. In industrial countries it is one of the most frequent causes of anaemia and can be a cause of labour absenteeism. Facing a patient with bleeding in the Accident and Emergency Service, the first thing is to evaluate the haemodynamic repercussion and take the necessary measures. A detailed case history and exploration are carried out to establish its origin. A uterine origin is usually considered when bleeding appears in the genital area, but this is not always the case, and it is necessary to rule out an origin in the lower genital tract, upper genital tract or an extragynaecological origin in the digestive and/ or urological area. With the diagnosis an appropriate treatment will be considered, as well the possible need for hospital admission.
\end{abstract}

Key words. Uterine bleeding. Dysfunctional uterine haemorrhage. Gynaecological emergencies.
Servicio de Obstetricia y Ginecología. Hospital Virgen del Camino. Pamplona

\section{Correspondencia:}

Izarbe Jiménez Ubieto

Francisco Javier Sáenz de Oiza, 34-bajo A

31012 Pamplona

E-mail: Izarbeju@terra.es 


\section{INTRODUCCIÓN}

El sangrado genital es la alteración ginecológica más frecuente en las mujeres en edad reproductiva siendo, en los países occidentales, una de las causas más frecuentes de anemia. En ocasiones puede motivar absentismo laboral ${ }^{1}$. Para entender el tema se deben tener claros una serie de conceptos en relación a los diferentes tipos de sangrado que se pueden encontrar en una consulta de urgencias de ginecología. Habrá que diferenciar el sangrado uterino normal que es la menstruación, producida por la descamación del endometrio, tras la deprivación hormonal, con una periodicidad entre 22 a 40 días, una cantidad de 35-45 ml/ciclo y una duración menor de 7 días, de los patrones de sangrado anormales. Estos sangrados anormales se clasifican según sea su presentación con respecto al ciclo menstrual y se definen con diferentes términos. La metrorragia es un sangrado a intervalos irregulares, de cantidad variable sin relación con el ciclo menstrual. La menorragia es un sangrado menstrual de características cíclicas, cuya cantidad supera los $80 \mathrm{ml} /$ ciclo. La polimenorrea se produce cuando existen sangrados menstruales con intervalos menores a 22 días. La hipermenorrea son intervalos cíclicos de una duración mayor a 7 días. El sangrado intermenstrual se produce entre menstruaciones normales. Por último la menometrorragia es un sangrado de intensidad y duración aumentada, con intervalos irregulares, aunque debe tenerse en cuenta que esta nomenclatura no es unánimemente admitida.

\section{ETIOLOGÍA}

Ante un sangrado habrá que tener en cuenta la posibles causas para poder orientar el diagnóstico de una forma precisa ${ }^{2,3}$ (Tabla 1).

Tabla 1. Causas de sangrado genital.

\begin{tabular}{|c|c|c|c|}
\hline $\begin{array}{l}\text { Complicaciones } \\
\text { de la gestación }\end{array}$ & Orgánicas & Sistémicas & $\begin{array}{c}\text { Hemorragia uterina } \\
\text { disfuncional }\end{array}$ \\
\hline Amenaza de aborto & $\begin{array}{l}\text { Extraginecológicas } \\
\text { (uretra, vejiga, recto) }\end{array}$ & $\begin{array}{l}\text { Fármacos } \\
\text { (ACHO, ACO, } \\
\text { corticoides, TMX) }\end{array}$ & Ovuladora \\
\hline Aborto incompleto & $\begin{array}{l}\text { Vulva (atrofia, } \\
\text { traumatismo, infección, } \\
\text { endometriosis, tumores) }\end{array}$ & $\begin{array}{l}\text { Alteraciones de la } \\
\text { coagulación }\end{array}$ & Anovuladora \\
\hline Aborto en curso & $\begin{array}{l}\text { Vagina (atrofia, } \\
\text { traumatismo, infección, } \\
\text { cuerpo extraño, tumor) }\end{array}$ & Enfermedad hepática & \\
\hline Gestación ectópica & $\begin{array}{l}\text { Cérvix (ectopia, pólipo, } \\
\text { POP; endometriosis, } \\
\text { infección, tumor) }\end{array}$ & Enfermedad renal & \\
\hline Enfermedad trofoblástica & $\begin{array}{l}\text { Útero (atrofia, } \\
\text { endometritis, DIU, } \\
\text { adenomiosis, mioma, } \\
\text { pólipos, carcinoma) } \\
\text { Trompas } \\
\text { Ovarios (rotura de quiste, } \\
\text { tumor) }\end{array}$ & $\begin{array}{l}\text { Endocrinos: tiroides, } \\
\text { Cushing, GnRH, PRL } \\
\text { Cardiopatía }\end{array}$ & \\
\hline
\end{tabular}

La causa más habitual de sangrado o desviación de una menstruación regular y predecible es una complicación de la gestación, por tanto la posibilidad de embara- 
zo debe descartarse como primera posibilidad en la consulta de urgencias ante un sangrado anormal.

Frecuentemente se encuentra un origen orgánico como miomas, pólipos, neoplasias endometriales y/o cervicales o sangrados de origen genital diferente del uterino incluyendo, traumatismos genitales, cuerpos extraños fácilmente observables con la exploración. En estos casos las pacientes suelen tener ciclos regulares con síndrome premenstrual, cuya duración y/o cantidad está aumentada o existe manchado intermenstrual.

En ocasiones los ciclos menstruales irregulares puede ser uno de los primeros síntomas de un trastorno tiroideo. Otras veces se deben a patología sistémica como la posibilidad de una coagulopatía, enfermedades graves renales y/o hepáticas, toma de fármacos (anticoncepción hormonal, anticoagulantes, tamoxifeno...).

La hemorragia uterina disfuncional (HUD) será un diagnóstico de exclusión tras descartar las posibles etiologías nombradas anteriormente. La HUD se define como una hemorragia anormal que procede de útero en ausencia de patología orgánica y de gestación. El origen de la producción de la HUD es, en muchos casos, difícil de identificar, existiendo causas endocrinas o debidas a factores locales uterinos. Desde el punto de vista endocrino la HUD se debe a una alteración del eje hipotálamo-hipófisis-ovario, que provoca una secreción anómala de los esteroides ováricos causando sangrado por deprivación o por disrupción. La hemorragia por deprivación se debe a la supresión o disminución brusca de la acción de las hormonas ováricas sobre el endometrio provocando su descamación. La disrupción es el mecanismo más frecuente en las HUD y se produce por la acción mantenida durante largo tiempo de las hormonas ováricas sobre el endometrio. Por otro lado, la HUD puede estar producida por factores locales uterinos ya que existen mecanismos locales relacionados con la alteración tales como el tono vascular uterino, la hemostasia y la regeneración del endometrio, que pueden ser el origen de hemorragia. A efectos clínicos las HUD se clasifican en ovulatorias y anovulatorios (Tabla 2).

Tabla 2. Clasificación de la hemorragia uterina disfuncional.

\begin{tabular}{|c|c|}
\hline Ovulatoria & Anovulatoria \\
\hline $15 \%$ de las HUD & HUD más frecuente \\
\hline En mujer madura entre $20-40$ años & Perimenopausia y pubertad \\
\hline $\begin{array}{l}\text { Alteraciones de la fase proliferativa, secretora o } \\
\text { ambas }\end{array}$ & $\begin{array}{l}\text { Estímulo continuo de estrógenos sin oposición de } \\
\text { la progesterona }\end{array}$ \\
\hline $\begin{array}{l}\text { Cíclicas y clínicamente cursan con polimenorrea, } \\
\text { spotting ovulatorio, spotting premenstrual, hiperme- } \\
\text { norrea y menorragias }\end{array}$ & $\begin{array}{l}\text { No cíclico de intensidad y duración variable. No se } \\
\text { acompaña de síndrome premenstrual. Cursan con } \\
\text { un sangrado generalmente abundante y tras un pe- } \\
\text { riodo de amenorrea de } 6-8 \text { semanas }\end{array}$ \\
\hline Causas & Causas \\
\hline $\begin{array}{l}\text { Fase folicular alargada } \rightarrow \text { endometrio excesiva } \\
\text { proliferación sin tej conectivo } \rightarrow \text { con progesterona } \\
\text { hemorragia por disrupción } \rightarrow \text { hipermenorrea }\end{array}$ & $\begin{array}{l}\text { Alteraciones eje hipotálamo -hipofisario- ovario: } \\
\text { perimenopausia, pubertad, estrés, alteraciones } \\
\text { bruscas de peso, actividad física intensa, obesidad, } \\
\text { ansiedad, psicofármacos }\end{array}$ \\
\hline $\begin{array}{l}\text { Alteraciones de los receptores } \rightarrow \text { maduración irre- } \\
\text { gular } \rightarrow \text { hipermenorreas y sangrados postmens- } \\
\text { truales }\end{array}$ & Administración exógena hormonas \\
\hline $\begin{array}{l}\text { Cuerpo lúteo insuficiente } \rightarrow \text { escasa maduración } \rightarrow \\
\text { sangrado premenstrual }\end{array}$ & $\begin{array}{l}\text { Patología endocrina (tiroideas, síndrome de ovario po- } \\
\text { liquístico, alteración suprarrenal, hiperprolactinemia) }\end{array}$ \\
\hline $\begin{array}{l}\text { Enfermedad de Halban (cuerpo lúteo persistente) } \rightarrow \\
\text { sangrado prolongado }\end{array}$ & Alteraciones hepáticas y / o renales \\
\hline
\end{tabular}




\section{DIAGNÓSTICO}

Una anamnesis detallada es el instrumento aislado más útil para el diagnóstico diferencial del sangrado. Es muy importante porque es posible la obteción de unos datos que nos orientarán hacia la gravedad y etiología del sangrado para posteriormente aplicar un tratamiento correcto.

El primer dato a tener en cuenta es la $\operatorname{edad}^{4}$ de la paciente ya que existen causas más frecuentes de sangrado en cada periodo de la vida. Por ejemplo, en la perimenopausia o postmenarquia precoz siempre habrá que pensar en la anovulación como posible etiología, en cambio en la menopausia la causa más frecuente es la atrofia, pero habrá que descartar un cáncer por su elevada prevalencia (en el 10\% de los sangrados). En una paciente joven con sangrados abundantes habrá que descartar una diatesis hemorrágica ${ }^{5}$ (Tabla 3 ).

Tabla 3. Causas más frecuentes de sangrado por grupo de edad.

\begin{tabular}{|c|c|c|c|c|}
\hline Premenarquia & $\begin{array}{c}\text { Post menarquia } \\
\text { precoz }\end{array}$ & Años reproductivos & Perimenopausia & Postmenopausia \\
\hline Cuerpo extraño* & Anovulación* & Anovulación* & Anovulación* & Atrofia* \\
\hline Traumatismo & Embarazo & Embarazo & Orgánica & Cáncer (10\%) \\
\hline Infección & $\begin{array}{l}\text { Diatesis } \\
\text { hemorrágica }\end{array}$ & Cáncer & Cáncer & $\mathrm{TSH}$ \\
\hline Prolapso uretral & Infección & Orgánica & & \\
\hline Sarcoma Botrioides & & Infección & & \\
\hline Tumor de ovario & & $\begin{array}{l}\text { Alteraciones } \\
\text { endocrinas }\end{array}$ & & \\
\hline Pubertad precoz & & $\begin{array}{l}\text { Diátesis } \\
\text { hemorrágica }\end{array}$ & & \\
\hline & & Fármacos & & \\
\hline
\end{tabular}

* En cursiva la causa más frecuente en cada grupo de edad.

En la anamnesis se debe prestar atención a los antecedentes familiares, sobre todo de cáncer de endometrio, colon, ovario y mama. De los antecedentes personales es preciso analizar las alergias, antecedente de cirugías (recientes o no), existencia de patología extraginecológica como la presencia de hemorragias (epistaxis, gingivorragias), hematomas frecuentes, petequias (todas ellas harán pensar en una causa sistémica del sangrado como problemas de coagulación primarios o secundarios a patología renal, hepática u otra) taquicardias, síntomas vasomotores o si está tomando tratamiento que podría causarle el sangrado como psicotropos, anticoagulantes, corticosteroides, citos- táticos, hábitos tóxicos... Los antecedentes personales ginecológicos a tener en cuenta son menarquia, fecha de la última menstruación, características de los últimos ciclos menstruales, fórmula menstrual (estos tres últimos datos nos orientarán a un sangrado en relación con una gestación y nos obligarán a solicitar una prueba de embarazo), fórmula obstétrica y fecha de la última gestación (aborto o parto), actividad sexual, método anticonceptivo que utiliza actualmente: natural, barrera, anticoncepción hormonal (ACH) (tipo), dispositivo intrauterino -DIU- (tipo), implante, esterilización tubárica. Aunque lo que más ayudará en el diagnóstico son los datos relacionados con el proceso actual (Fig. 1). 


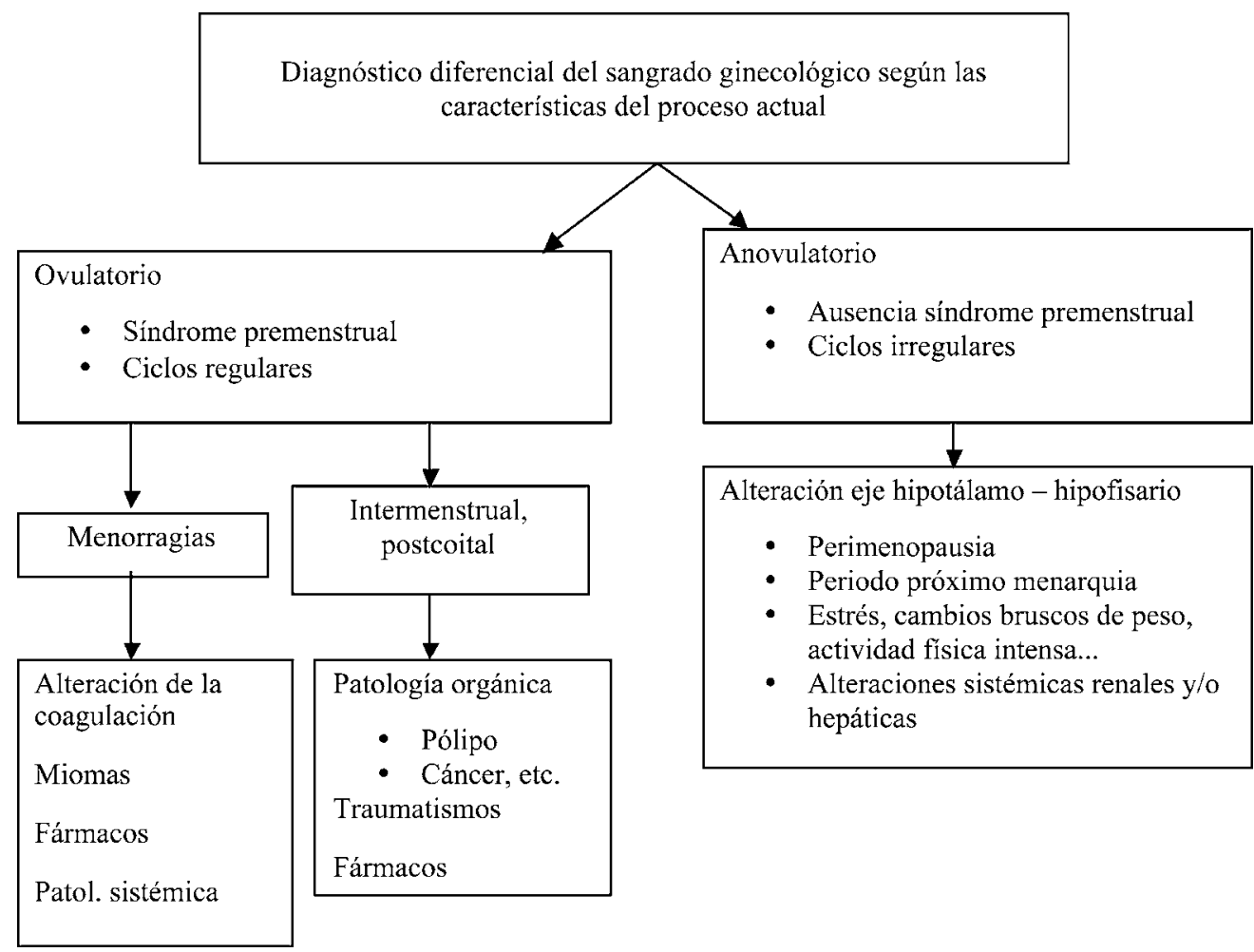

Figura 1. Diagrama de diagnóstico diferencial del sangrado ginecológico.

Se realizará una valoración del sangrado tanto de la cantidad, inicio, frecuencia, duración, relación con actividades como el coito... En un sangrado en relación con el coito habrá que pensar en patología cervical, buscar desgarros... En sangrados irregulares es importante valorar si se acompañan de síntomas premenstruales para evaluar si es un sangrado ovulatorio o anovulatorio; la existencia de factores precipitantes como un trauma. Indagar si ha tenido antecedentes de procesos similares. Buscar síntomas acompañantes como dolor y su localización, fiebre, leucorrea, disuria, alteraciones digestivas (vómitos, alteraciones del ritmo intestinal). Situaciones personales y antecedentes personales (Tabla 4).
Tabla 4. Valoración de la cantidad del sangrado menstrual.

\begin{tabular}{ll}
\hline \multicolumn{1}{c}{ Criterio } & \multicolumn{1}{c}{ Regla normal } \\
\hline $\begin{array}{l}\text { Frecuencia cambio } \\
\text { compresa }\end{array}$ & Cada más de 3 horas \\
$\begin{array}{l}\text { Número de compresas/ } \\
\text { menstruación }\end{array}$ & $\begin{array}{l}\text { Menor de 21/ } \\
\text { menstruación } \\
\text { Cambio de compresa } \\
\text { por la noche }\end{array}$ \\
$\begin{array}{l}\text { Produce anemia } \\
\text { No }\end{array}$ & No \\
\hline
\end{tabular}

Para clarificar la etiología del sangrado es importante el examen físico de la paciente. Analizar en primer lugar la repercusión 
clínica que produce el sangrado para ver si es preciso la estabilización hemodinámica previo a continuar la anamnesis y exploración, mediante la valoración de las constantes vitales (tensión arterial, frecuencia cardiaca y temperatura). En una paciente taquicárdica e hipotensa previamente comenzaremos con sueroterapia e intentar ceder el sangrado. Si no, comenzar con el resto de la exploración.

Realizaremos una exploración general en la que la presencia de hematomas, hirsutismo y obesidad puede orientar hacia un origen sistémico del sangrado. Por otro lado, el tamaño del tiroides, puede orientar hacia un hipertiroidismo. Estos hallazgos requerirán un seguimiento posterior más profundo bien en el ingreso, bien en consulta externa. En la exploración abdominal buscaremos la localización de posibles puntos dolorosos, irritación peritoneal, presencia de masas, ascitis...ya que a veces cuadros infecciosos pueden dar spotting, los quistes de ovario persistente cursan también con sangrado.

Finalmente se realizará una exploración ginecológica para establecer el origen del sangrado, que no siempre es de origen uterino. Podrá ser extrauterino, debiendo valorarse mediante inspección de genitales externos, vulva, recto y uretra. Con espéculo se valorará vagina y exocervix. Es preciso una búsqueda de lesiones por atrofia, traumatismos, infecciones, cuerpos extraños y/o tumores. Se completa el examen con tacto bimanual, e intentaremos identificar su etiología: obstétrica, orgánica, hematológica, oncológica, disfuncional (con o sin ovulación), mediante estudios diagnósticos complementarios. Durante la exploración ginecológica también se verá la cantidad de sangrado.

Una vez realizada la anamnesis y la exploración física el diagnóstico estará más o menos orientado y se debe continuar con una serie de pruebas tales como el laboratorio, el estudio ecográfico y la biopsia para confirmar el diagnóstico.

En toda mujer en edad fértil, siempre es obligado descartar un posible embarazo con un test de gestación en orina. Si es un sangrado moderado-abundante se realizará un hemograma completo con plaquetas y estudio de coagulación. Con la hemoglobina y hematocrito valoraremos la severi- dad del sangrado, a posteriori se podrá hacer un nivel de ferritina que orientará hacia pérdidas hemáticas crónicas. El estudio de coagulación es importante sobre todo en pacientes jóvenes, con sangrados anormales extragenitales y menorragias por la posibilidad de diátesis hemorrágicas. En caso de la aparición de la fórmula leucocitaria alterada orientará en casos de infección acompañante, que podría asociarse a cultivo cervical para la detección de Clamidias y/o gonococo. En caso de sospechar patología acompañante podrán pedirse pruebas de tiroides, función hepática y/o renal que se manejará desde el ingreso o a posteriori en su ginecólogo según la gravedad del proceso.

La ecografía pélvica, sobre todo vía transvaginal ${ }^{6}$, es una técnica de gran utilidad en el diagnóstico ginecológico de patología tanto orgánica como funcional. Dentro de la patología orgánica podrá ser intrauterina o extrauterina y se encuentran diferentes causas: uterina como miomas, adenomiosis, localización de la gestación; patología endometrial (pólipos, hiperplasias endometriales, cáncer de endometrio, dispositivo intrauterino...); patología ovárica: quistes funcionales, tumoraciones malignas...Otra patología pélvica: masas pélvicas de origen no ginecológico (vesicales, rectal...). Ante la exclusión de patología orgánica la valoración del estado endometrial así como las evidencias del funcionamiento ovárico son útiles para la elección del tratamiento.

Se biopsiarán las lesiones sospechosas que se encuentren durante la exploración en vulva, vagina, cérvix. Para la realización de una biopsia de endometrio previamente siempre hay que descartar la posibilidad de gestación y de infección pélvica actual. Se debe realizar biopsia de endometrio en toda mujer mayor de 35 años. En determinadas circunstancias también puede ser conveniente realizarla en menores de 35 años como cuando existe historia familiar o personal de cáncer de endometrio, ovario, mama o colon, si la paciente está en tratamiento con tamoxifeno o terapia estrogénica, en casos de anovulación crónica, obesidad, diabetes, antecedente personal de hiperplasia endometrial y si existen marcadores ecográfico y/o clínicos como sangrado persistente (Tabla 5). 
Tabla 5. Indicaciones de biopsia endometrial en mujeres menores de 35 años.

Historia familiar o personal de cáncer de

endometrio, ovario, mama o colon

Uso de tamoxifeno o terapia estrogénica

Anovulación crónica

Obesidad

Antecedente de hiperplasia endometrial

Diabetes

Si existen marcadores ecográficos y/o clínicos (ejemplo sangrado persistente)

\section{TRATAMIENTO}

Como regla general para el tratamiento de la hemorragia será preciso: cohibir la hemorragia, tratar la anemia si existe y regularizar el ciclo, si está indicado y evitar recidivas. En la consulta de urgencias solucionaremos el proceso hemorrágico agudo, posteriormente se establecerá un tratamiento a corto o medio plazo para evitar recidivas o controlar el ciclo con control fuera de la urgencia.

Para cumplir el primer objetivo de cohibir la hemorragia, habrá que valorar la cantidad y causa del sagrado porque unos precisarán ingreso y otros no. Serán causas de indicación de ingreso hospitalario, entre otras: sangrado agudo muy abundante: hemoglobina $<9 \mathrm{~g} / \mathrm{L}$, signos de hipovolemia, hipotensión, taquicardia; sospecha de coagulopatías; sangrados importantes asociados a patología de la gestación; patología orgánica uterina que precisa tratamiento quirúrgico urgente; proceso séptico en fase aguda, fiebre, alteración de la formula leucocitaria. Se instaurará terapia específica con antibioticoterapia IV; en caso de sospecha de neoplasia ginecológica maligna, se agilizarán las pruebas necesarias para instaurar el tratamiento médico-quirúrgico específico.

En los casos en que exista inestabilidad hemodinámica el tratamiento inicial se administrará bajo estricto control médico, a fin de lograr estabilizar hemodinámicamente a la paciente, monitorización, aporte de volumen, iones, transfusión si precisa, etc.

Simultáneamente al tratamiento general de la fase aguda, el objetivo será detener la hemorragia, mediante tratamiento médico o quirúrgico específico en cada caso ${ }^{7,8}$.
En el caso de sangrados muy abundantes podrán beneficiarse de un tratamiento médico y/o quirúrgico agresivo. El tratamiento hormonal consistirá en administrar por vía IV estrógenos equinos conjugados, $20 \mathrm{mg} / 4-6 \mathrm{~h}$, durante las primeras 24 horas y, para evitar la hemorragia por deprivación, se continúa por vía oral con un preparado de estrógeno-progestágeno tipo anovulatorio, durante 12-15 días. Posteriormente se instaurará un tratamiento a medio plazo que va a depender de las características de la paciente. Se beneficiarán de este tratamiento las metrorragias y /o menorragias con endometrio escaso ya que los estrógenos servirán para ayudar a proliferar el endometrio, crear receptores de progesterona y beneficiarse de su acción hemostática En este caso el tratamiento quirúrgico (legrado) sería contraproducente porque podría aumentar aun más la hemorragia, aunque sí estaría aceptado la cohibición de la hemorragia mediante la introducción de una sonda de Foley intracavitaria ${ }^{9}$.

Por otro lado, existe la posibilidad de un tratamiento médico con antifibrinolíticos más ampliamente aceptado en nuestro medio. Las posibilidades son el acido tranexámico (Amchafibrim ${ }^{\circledR}$ ): 0,5-1 g / 8 horas iv cuyos efectos secundarios son mínimos (ocasionalmente vómitos y diarrea) y el riesgo tromboembólico en los estudios realizados no parece se superior a los de la población control de la misma edad. Y el ácido aminocaproico (Caproamin -Fides ${ }^{\circledR}$ ): 4-5 g en $250 \mathrm{ml} \mathrm{SF} \mathrm{o} \mathrm{G} 5 \%$ en una hora hasta 8 horas o cese del sangrado.

Si la causa de la hemorragia es extrauterina como traumatismo genital, úlcera, dislaceración, etc. que no puede ser solucionado en la consulta de urgencias podría requerir la reparación quirúrgica.

Si la hemorragia uterina es muy intensa y aguda o no cede con tratamiento médico o la paciente no es susceptible de dicho tratamiento estaría indicado el tratamiento quirúrgico. En estos casos es útil la valoración ecográfica del endometrio ya que ante un sangrado muy abundante con inestabilidad hemodinámica que precise cohibición precoz, presenta un endometrio fino y no fuera candidata a tratamiento hormonal podría valorarse el taponamiento uterino mediante una sonda vesical, introduciendo la sonda e inflando el globo en cavidad ute- 
rina y un endometrio grueso precisará de legrado uterino completo En casos excepcionales si fallan todas las medidas anteriores podría recurrirse a una histerectomía.

Por otro lado están los sangrados más moderados, que no precisan ingreso y el tratamiento podrá ser menos agresivo. Para empezar se intentará un tratamiento etiológico. Por ejemplo, en caso de diagnóstico de patología orgánica como exéresis de pólipos endocervicales con posterior tratamiento hemostático local, electrocoagulación, o lesionas canal genital que se solventarán, es posible la histeroscopia diagnóstica-terapéutica, que no precisa anestesia general, como resección de pequeños pólipos endometriales, biopsia dirigida de lesiones endometriales... Para el control de la hemorragia en estas patologías será necesario añadir tratamiento médico posterior hormonal y/o no hormonal (antifibrinolíticos, inhibidores de las PG). En los casos de patología orgánica que precisen tratamiento quirúrgico posterior se instaurará tratamiento para la hemorragia aguda y se indicará el seguimiento y tratamiento específico (quirúrgico o médico). En los cuadros infecciosos, como vaginitis, cervicitis, endometritis, enfermedad inflamatoria pélvica, en fase no aguda, que puede acompañar a la metrorragia, se instaurará el tratamiento específico local y/o general. Si el origen del sangrado se debe a patología sistémica se instaurará tratamiento médico como HUD y el tratamiento específico de la patología subyacente se establecerá en el momento o en consultas posteriores si precisa de más estudios. El sangrado también puede tener un origen iatrogénico con el uso de anticoncepción hormonal oral en cuyo caso deberemos asegurarnos del correcto uso de la anticoncepción. Si no lo toma adecuadamente habrá que educarle; si lo toma adecuadamente podrá valorarse cambiar la dosis a un preparado de mayor contenido estrogénico aumentando 2-3 comprimidos/día con el mismo anovulatorio e iniciar el siguiente ciclo con un anticonceptivo hormonal oral de mayor dosis. Cuando consideremos que el sangrado es secundario al DIU lo retiraremos y plantearemos otro método. Una opción en el tratamiento de la HUD es el DIU de levonorgestrel.

Una vez descartada otra patología pueden seguirse unas pautas para cohibir la hemorragia uterina. El tratamiento puede realizarse con los siguientes fármacos y mé- todos: gestágenos, gestágenos más estrógenos, estrógenos, antifibrinolíticos (vía oral).

Los gestágenos inducen transformación secretora de un endometrio proliferativo pudiendo originar una hemorragia por deprivación de forma autolimitada y predecible en ciclos anovulatorios con episodios de hemorragia intensa o prolongada. En caso de hemorragias intensas o prolongadas se ha recomendado la administración de dosis altas de gestágenos como $5-10 \mathrm{mg}$ (acetato de noretindrona) ó $10 \mathrm{mg}$ (acetato de medroxiprogesterona) cada 4 horas durante 24 horas o hasta el cese de la hemorragia, disminuyendo después la dosis 4 veces al día 4 días, 3 veces al día 3 días y 2 veces al día 2 semanas tras las que la mujer tendrá una hemorragia por deprivación. Después se instaura tratamiento para evitar nuevos episodios (3-6 meses). Asociación de estrógenos más gestágenos: en hemorragias prolongadas en mujeres jóvenes: 2 comprimidos diarios de baja dosis durante 7 días (aunque el sangrado cese en las primeras 24-48 horas), después se producirá un sangrado por deprivación, continuar con la toma diaria de un comprimido 21 días o 28 según preparado. Si se continúa la toma diaria de comprimidos seguido de los 2 comprimidos/día se evita la hemorragia hasta terminar la caja. En hemorragias intensas tomará 1 comprimido cada 4 horas 24 horas o hasta cese del sangrado. Después 4 comprimidos/día 4 días, 3 comprimidos/día 3 días y 2 comprimidos/día 2 semanas. Tras eso tendrá una hemorragia por deprivación que deberá ir seguida de tratamiento con gestágenos o anticoncepción hormonal 3-6 meses.

Administrar estrógenos en ocasiones en que el endometrio está muy adelgazado o incluso atrófico y los gestágenos no sean eficaces. Puede ser en situaciones de manchado vaginal intermitente o tras episodios prolongados de hemorragia intensa que dejan un tejido escaso residual. Estrógenos conjugados vía oral 1,25-2 mg de estradiol/4 horas durante 24 horas seguido de 1,25 mg/día de estrógenos conjugados ó $2 \mathrm{mg}$ de estradiol diario 7-10 días. Se deben asociar gestágenos los 5 últimos días y posteriormente se producirá una hemorragia por deprivación, luego continuar con tratamiento con gestágenos o anticonceptivos 3-6 meses.

Tras conseguir el cese de la hemorragia aguda hay que establecer un tratamiento 
de mantenimiento que controle los sangrados de los siguientes ciclos. Como medida general, en todos los casos, siempre hay que normalizar los depósitos de hierro y controlar la anemia que estas pacientes suelen presentar. Posteriormente el tratamiento se individualizará según la edad, deseos genésicos, antecedentes personales y familiares... de la paciente, pudiendo ser médico y/o quirúrgico.

En este sentido sería interesante tener en cuenta el uso de AINES. Reducen el contenido de prostaglandinas en el endometrio reduciendo la pérdida sanguínea en las hemorragias ovulatorias y en las incrementadas que se relacionan con DIU, además de aliviar la dismenorrea y la cefalea. Los más usados son el naproxeno e ibuprofeno, durante los 2-3 primeros días de la menstruación.
Los antifibrinolíticos tienen un papel interesante en las menorragias administrándolos vía oral los 2-3 primeros días del ciclo (500 $\mathrm{mg} / 8$ horas).

Las pautas de tratamiento hormonal tras cese de la hemorragia aguda serían los gestágenos o los combinados de estrógenos y progesterona.

Gestágenos como acetato de medroxiprogesterona (Progevera ®) 10-20 mg /día durante 10-14 días comenzando en el día 14 del ciclo, a lo largo de 3-6 meses o acetato de noretindrona.(Aygestin $\left.{ }^{\circledR}\right) 10 \mathrm{mg} /$ día durante 10-14 días comenzando en el día 14 del ciclo, a lo largo de 3-6 meses.

Combinados de estrógenos y progesterona $(\mathrm{ACH})$ por vía oral, transdérmica o vaginal durante 3-6 meses.

Se propone un protocolo de actuación ante un sangrado en urgencia (Fig. 2).

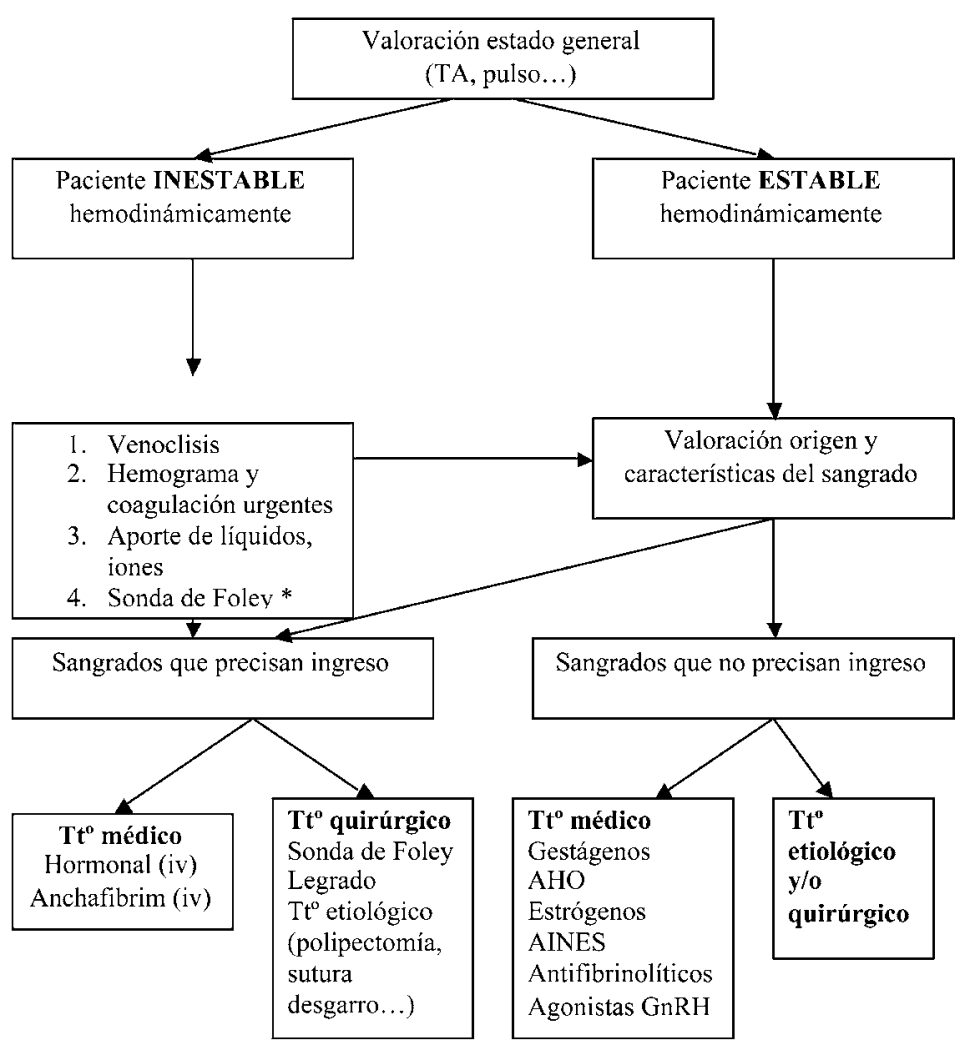

* En ocasiones si el sangrado es de origen uterino y la paciente esta inestable puede colocarse una sonda intrauterina cohibiendo la hemorragia hasta conseguir estabilizar a la paciente.

Figura 2. Protocolo de actuación ante sangrado de origen ginecológico. 


\section{BIBLIOGRAFÍA}

1. Martín MJ, Tasende M, Iglesias E. Metrorragia en la edad adulta. En: Cañete Palomo, María Luisa; Cabero Roura, Luis. Urgencias en Ginecología y Obstetricia. Aproximación a la medicina basada en la Evidencia. Edita FISCAM. 2007: 525-546.

2. HATASAKA $H$. The evaluation of abnormal uterine bleeding. Clin Obst Gynecol 2005: 48: 258-273.

3. BRENNER PF. Differential diagnosis of abnormal uterine bleeding. Am J Obstet Gynecol 1996; 175: 766-769.

4. Philipp CS, Faiz A, Dowling N, Dilley A, Michaels LA, Ayers C, Age and the prevalence of bleeding disorders in women with menorrhagia. Obstet Gynecol 2005; 105: 61-66.

5. Demers C, Derzko C, David M, Douglas J. Gynaecological and Obstetric Management of Wo- men with Inherited Bleeding Disorders. SOGC Clinical practice guidelines 2005:163.

6. Goldstein SR, Zeltser I, Horan CK, SNyder JR, SCHWARTZ LB. Ultrasonography-based triage for perimenopausal patients with abnormal uterine bleeding. Amer J Obstet Gynecol 1997; 177: 102-108.

7. Dysfunctional Uterine Bleeding: Treatment \& Medication. BeHERA MA, PRICE TM. Disclosures Updated: 2008: 30.

8. Speroff L, Fritz MA. Hemorragia uterina disfuncional. En: Endocrinología Ginecológica Clínica y Esterilidad. $7^{\underline{a}}$ Edición en inglés. $2^{\text {a }}$ Edición en español. Edita Lippincott, Williams \& Wilkins. 2006: 547-572.

9. Hoffman MK, Meilstrup JW, Shackelford DP, KAMINSKI PF. Arteriovenous malformations of the uterus: an uncommon cause of vaginal bleeding. Obstet Gynecol Surv 1997; 52: 736-740. 